\title{
Enhanced Solubility and Dissolution Rate of Clopidogrel by Nanosuspension: Formulation via High Pressure Homogenization Technique and Optimization Using Box Behnken Design Response Surface Methodology
}

\author{
Mohd Javed Qureshi ${ }^{a^{*}}$, Fung Fuie Phin ${ }^{\mathrm{a}}$, Sreenivas Patro ${ }^{\mathrm{b}}$ \\ ${ }^{a}$ Department of Dosage Form Design, Faculty of Pharmacy, MAHSA University 41200, Jln SP 2/7, Bandar Saujana Putra, Jenjarom, Se langor, Malaysia. \\ ${ }^{b}$ Department of Pharmaceutical Technology, School of Pharmacy, Taylors University Lake side campus, Subang Jaya, Selangor Darul Ehsan, Malaysia.
}

\begin{tabular}{|c|c|}
\hline ARTICLE INFO & ABSTRACT \\
\hline Article history: & \multirow{9}{*}{$\begin{array}{l}\text { The present study was aimed to formulate nanosuspensions using high pressure homogenization (HPH), a top } \\
\text { down technique for enhancement of dissolution rate and solubility of clopidogrel using Pluronic F127 as } \\
\text { stabilizer. Clopidogrel is categorized as a BCS class II agent having oral bioavailability less than } 50 \% \text {. The } \\
\text { formulation scheme was generated by Box- Behnken design of response surface methodology. The formulated } \\
\text { nanosuspensions were assessed on particle size, polydispersity index, and zeta potential. Three formulations } \\
\text { were selected based on different predicted particle size with manipulation of parameters using response } \\
\text { optimizer. The selected formulations were checked on percentage of bias in between predicted value and } \\
\text { observed value and evaluated based on drug content, drug entrapment efficiency and in vitro dissolution study. } \\
\text { The formulation was optimized based on the smallest particle size and highest percentage of in vitro cumulative } \\
\text { drug release. Formulation FF3 was selected as optimized formulation which attributed to the smallest particle } \\
\text { size }(478.1 \mathrm{~nm}) \text { and the highest } \% \mathrm{CDR} \text { in both } 0.1 \mathrm{~N} \mathrm{HCl}(98.37 \%) \text { and phosphate buffer ( } 48.67 \%) \text {. The } \\
\text { optimized formulation has shown a significant } 2 \text { folds enhancement in dissolution rate in } 0.1 \mathrm{~N} \mathrm{HCland} 10 \text { folds } \\
\text { improvement in pH } 6.8 \text { phosphate buffers with } 0.1 \% \mathrm{w} / \mathrm{v} \text { Tween } 80 \text { compared to pure drug suspension. }\end{array}$} \\
\hline Received on: $30 / 08 / 2016$ & \\
\hline Accepted on: & \\
\hline Available or & \\
\hline Key words: & \\
\hline Nanosuspension, & \\
\hline Clopidogrel, Pluronic F127, & \\
\hline Box- Behnken, In Vitro & \\
\hline Dissolution. & \\
\hline
\end{tabular}

\section{INTRODUCTION}

The ease of oral administration make it become a more preferential route for patients to consume medication (Kumar and Singh, 2013). However, most of the available active pharmaceutical ingredients are poorly water soluble which have dissolution limitation in gastrointestinal (GI) tract and eventually causing poor oral bioavailability issue that will greatly reduce therapeutic effectiveness of the drug pharmacologically, resulting in compromised patient adherence problem (Shid et al., 2013; Chen et al., 2011). Therefore, the improvement of dissolution rate and saturation solubility is essential to achieve optimum oral

\footnotetext{
* Corresponding Author

Mohd Javed Qureshi, Department of Dosage Form Design, Faculty of Pharmacy, MAHSA University 41200, Jln SP 2/7, Bandar Saujana

Putra, Jenjarom, Selangor, Malaysia. Email: javed @ mahsa.edu.my
}

bioavailability. Based on the Noyes-Whitney equation, dissolution rate and saturation solubility of poor water soluble drug can be enhanced through size reduction into nano-range due to the increase of interfacial surface area available for wetting (Liu et al., 2010). Hence, in recent years, nanotechnology has become a promising approach for the formulation of poorly water soluble drug (Attari et al., 2015). Nanosuspension is one of the available nanoformulations that are effective to solve the solubility related bioavailability problems (Patel and Agrawal, 2011). It is defined as solid dispersion of active pharmaceutical ingredients in size of smaller than $1 \mu \mathrm{m}$ in a liquid vehicle with addition of surfactant for stabilization purpose (Yadav and Singh, 2012). Nanosuspension can be prepared by either top-down technique which is more mechanical-effective and scalable or bottom-up technique which is more cost and time-saving in nanoparticles production (Liu et al., 2010). 
High pressure homogenization (HPH) is a method categorised under top down technology by involving the reduction of particle size from larger size to the range of size in nm (Mane $e t$ al., 2014). HPH was selected in the nanosuspension production of this study due to its superiority in minimised variation between batch to batch after the process parameters are adjusted to optimum which can result in high reproducibility in the production of nanosuspension and its ability to produce nanosuspension in a wide range of concentration (Jagdale et al., 2010). In this study, clopidogrel was the selected drug to be prepared by HPH method for the production of nanosuspension. Clopidogrel is an antiplatelet agent which is widely used in prevention of thrombotic event in cardiovascular disease. Upon administered, clopidogrel is rapidly absorbed in upper GI tract, metabolized by cytochrome P450 to its active thiol metabolite, which has a short elimination half-life (1hour). Furthermore, bioavailability of clopidogrel is also predicted to be generally low, which is approximate to $50 \%$ according to volume of excreted unchanged drug and metabolite presented in urine and feces. According to the biopharmaceutics classification system (BCS), Clopidogrel is categorized as a class II agent having poor water solubility and high permeability, which is responsible for its poor oral bioavailability (Takagi et al., 2006). Besides that, its highly $\mathrm{pH}$ dependent absorption characteristic at acidic $\mathrm{pH}$ is another factor of causing poor oral bioavailability as the drug gets precipitated when it reaches small intestine which has basic environment (Raghuvanshi and Pathak, 2014). The poor oral bioavailability and short elimination half-life of clopidogrel leads to the need of higher dose and more frequent dosing in order to achieve the desired therapeutic effect (Shahba et al., 2012). However, extra doses increase the risk of drowsiness, dizziness, hypotension, and aggravation of extrapyramidal symptoms, lupus on skin and internal organs, and jaundice (Teva Pharmaceuticals Europe B.V., 2015; Takagi et al., 2006). Nanosuspension has shown to have greater drug loading compared to other oral dosage form, and thus, lower dose is required to achieve intended therapeutic effect and patient compliance can be improved with the use of this drug delivery system (Yadav and Singh, 2012; Rabinow, 2004). The aim of this work is to formulate the clopidogrel nanosuspension by high pressure homogenization method to improve its solubility and to find out the effect of Drug:stabilizer mass ratio, number of homogenization cycles and pressure on the formulation.

\section{METHODS}

\section{Materials}

Clopidogrel was obtained as a gift sample from Fleming Labs (India). Pluronic F127 was obtained as a gift sample from BASF (United States). All other reagents used were of analytical grades.

\section{Preparation of Nanosuspension}

The nanosuspension of clopidogrel was prepared by $\mathrm{HPH}$ method. Clopidogrel $(1 \mathrm{mg} / \mathrm{ml})$ was added into $70 \mathrm{ml}$ of mixed solution of Pluronic F-127 in varied stabilizer:drug mass ratio $(20 \%$ to $40 \% \mathrm{w} / \mathrm{w})$ with the assist of magnetic stirrer. The resulting mixture was further mixed using high speed homogeniser (UltraTurrax T 25 Digital, IKA, Germany) was applied at a speed of $10,000 \mathrm{rpm}$ for 15 minutes to form pre-suspension. Then, the presuspension was forced through a high pressure homogeniser (Microfluidics M110P-20K, Newton, Massachusetts, USA) under pressure applied within range of 800 bars to 1300 bars for 10 cycles then continued with remaining cycles (5-15 cycles) under 1500 bar. Some of the formulations was repeated at which the presuspension was forced through the high pressure homogeniser under pressure applied within range of 800 bars to 1300 bars for 10 cycles then continued with the same pressure for further 15 cycles.

\section{Experimental Design}

The formulation scheme of clopidogrel nanosuspension was generated using 15 runs, 3 factors, unblocked Box-Behnken design of Response Surface Methodology (RSM) in Minitab software (Minitab® 17.3.1 trial version, Sydney) by varying 3 parameters which included stabilizer:drug mass ratio in range of $20 \% \mathrm{w} / \mathrm{w}$ to $40 \% \mathrm{w} / \mathrm{w}$, homogenization pressure in range of 800 bar to 1300 bar in the first 10 cycles, and number of remaining homogenization cycle in the range of 5 to 15 cycles applied under 1500 bar as shown in Table 1.

The developed clopidogrel nanosuspensions were subjected for particle size analysis, polydispersity index (PDI), and zeta potential measurement.

Table 1: Formulations of clopidogrel nanosuspension generated using RSM.

\begin{tabular}{|c|c|c|c|c|c|}
\hline 氞 & 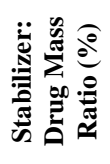 & 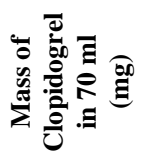 & 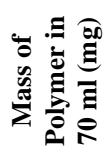 & 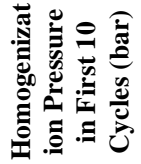 & 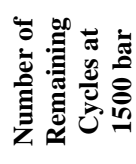 \\
\hline 1. & 40 & 70 & 28 & 800 & 10 \\
\hline 2. & 40 & 70 & 28 & 1050 & 15 \\
\hline 3. & 30 & 70 & 21 & 800 & 15 \\
\hline 4. & 20 & 70 & 14 & 1050 & 15 \\
\hline 5. & 30 & 70 & 21 & 1300 & 15 \\
\hline 6. & 40 & 70 & 28 & 1300 & 10 \\
\hline 7. & 30 & 70 & 21 & 800 & 5 \\
\hline 8. & 30 & 70 & 21 & 1050 & 10 \\
\hline 9. & 20 & 70 & 14 & 800 & 10 \\
\hline 10. & 20 & 70 & 14 & 1050 & 5 \\
\hline 11. & 30 & 70 & 21 & 1300 & 5 \\
\hline 12. & 30 & 70 & 21 & 1050 & 10 \\
\hline 13. & 20 & 70 & 14 & 1300 & 10 \\
\hline 14. & 30 & 70 & 21 & 1050 & 10 \\
\hline 15. & 40 & 70 & 28 & 1050 & 5 \\
\hline
\end{tabular}

\section{Response Analysis and Response Surface Analysis}

The result obtained for the 3 responses was entered into the worksheet of RSM and analysed using response analysis. The response that fitted to the model was selected based on the regression coefficient $\left(\mathrm{R}^{2}\right)$ value above $80 \%$ and $\mathrm{p}$-value below 0.05. Surface plot graphs were generated to determine the interaction effect of any 2 parameters on the selected response. 


\section{Selection and Optimization of Formulation}

Three formulations were selected based on 3 different predicted particle size with manipulation of parameters by using response optimiser. The produced nanosuspensions of the 3 selected formulations were checked for the percentage of bias based on particle size result obtained with the equation of the following:

\section{Percentage of Bias $(\%)=$ \\ Predicted value - Observed value $\times 100 \%$}

Observed value

The nanosuspensions of the 3 selected formulations were also evaluated on drug content, DEE, and in vitro dissolution study in order to find out the most optimized formulation in present study. The optimized formulation was further evaluated based on drug release kinetic study.

\section{Evaluation Test of Nanosuspension}

The evaluation tests were carried out to characterize the produced nanosuspension, including particle size and PDI analysis, zeta potential, drug content estimation study, DEE, in vitro dissolution study, and drug release kinetic study.

\section{Particle size and PDI analysis}

Particle size and PDI of the particles in the nanosuspension was measured by using Malvern Zetasizer (Model ZEN3600, Malvern, UK). A sample of nanosuspension was diluted with distilled water and subjected into disposable sizing cuvette for measurement at temperature of $25^{\circ} \mathrm{C}$ and scattering angle of $173^{\circ}$ with setting of dispersant refractive index (RI) at 1.33, material RI at 1.58 and material absorption at 1.000 in triplicates (Chiang et al., 2011; Agarwal and Bajpai, 2014).

\section{Zeta potential measurement}

The measurement of zeta potential was carried out using the additional electrode of Malvern Zetasizer that was used for particle size and PDI analysis. A sample of nanosuspension was diluted with distilled water and subjected into disposable sizing cuvette for measurement at temperature of $25^{\circ} \mathrm{C}$ with setting of dispersant RI at 1.33 and dielectric constant of dispersant at 78.5 in triplicates (Agarwal and Bajpai, 2014).

\section{Drug content estimation study}

An amount of $0.2 \mathrm{ml}$ of nanosuspension was dissolved in $20 \mathrm{ml}$ of $0.1 \mathrm{~N} \mathrm{HCl}$, filtered, and assayed using $\mathrm{UV}$ Spectrophotometer at $253 \mathrm{~nm}$ to determine the actual drug content in triplicates. The percentage of actual drug content was calculated based on the equation of the following:

Percentage of actual drug content $(\%)=$

$\frac{\text { Actual amount of drug }}{\text { Theoretical amount of drug }} \times 100 \%$

\section{Drug entrapment efficiency (DEE)}

An amount of $1.5 \mathrm{ml}$ of freshly prepared nanosuspension was centrifuged at $10,000 \mathrm{rpm}$ with for $30 \mathrm{~min}$ by using microcentrifuge. Then, $0.2 \mathrm{ml}$ of supernatant solution was diluted with $0.1 \mathrm{~N} \mathrm{HCl}$ and subjected to UV Spectrophotometer to measure the amount of drug which was unincorporated. The DEE is calculated by deducting the amount of free drug present in supernatant from the actual amount of drug present in the formulation. This test was carried out in triplicates. The DEE can be calculated based on the equation of the following:

$$
\begin{aligned}
& \text { Drug Entrapment Efficiency }(\mathrm{DEE} \%)= \\
& \frac{\text { Actual amount of drug - Amount of free drug }}{\text { Actual amount of drug }} \times 100 \%
\end{aligned}
$$

\section{In vitro dissolution study}

USP Type II dissolution apparatus was used to test the dissolution rate of the pure drug suspension of clopidogrel and nanosuspension by paddle method. The dissolution medium used for this study included $0.1 \mathrm{~N} \mathrm{HCl}$ and $\mathrm{pH} 6.8$ phosphate buffer with $0.1 \% \mathrm{w} / \mathrm{v}$ Tween 80 . A volume of dissolution medium was transferred to every vessel of the dissolution apparatus. The stirring rate was set at $50 \mathrm{rpm}$ and the temperature of dissolution media was set at $37 \pm 0.5^{\circ} \mathrm{C}$. Pure drug suspension and nanosuspension was transferred into one-end tied dialysis bag, then the open end of the bag was tied. The dialysis bag was attached on the paddle of dissolution apparatus. An amount of $5 \mathrm{ml}$ of sample was withdrawn at regular time interval. For $0.1 \mathrm{~N} \mathrm{HCl}$, the time intervals for sampling were $5 \mathrm{~min}, 10 \mathrm{~min}, 15 \mathrm{~min}, 20$ min, $30 \mathrm{~min}, 40 \mathrm{~min}$, and $60 \mathrm{~min}$ while for $\mathrm{pH} 6.8$ phosphate buffer with $0.1 \% \mathrm{w} / \mathrm{v}$ Tween 80 , the time intervals for sampling were $5 \mathrm{~min}, 10 \mathrm{~min}, 30 \mathrm{~min}, 50 \mathrm{~min}, 70 \mathrm{~min}, 90 \mathrm{~min}$, and $120 \mathrm{~min}$. An amount of $5 \mathrm{ml}$ dissolution medium was added in order to remain the sink condition. Sample was filtered, diluted and subjected to UV Spectrometer to determine the amount of drug released. The procedure was carried out in triplicates.

\section{Drug release kinetic study}

The release kinetic study of nanosuspension in $0.1 \mathrm{~N} \mathrm{HCl}$ and $\mathrm{pH} 6.8$ phosphate buffer with $0.1 \%$ w/v Tween 80 was evaluated based on 4 models, including zero order, first order, Higuchi model, and Korsmeyer-Peppas model and graphs were plotted for each model. In zero order, the graph of percentage of cumulative drug release (\% CDR) against time of dissolution was plotted. In first order, the graph of $\log \%$ CDR against time of dissolution was plotted. In Higuchi model, the graph of \% CDR against square root of time of dissolution was plotted. In Korsmeyer- Peppas model, the graph of $\log \%$ CDR against $\log$ of time of dissolution was plotted. The best fitted model was selected based on the highest $\mathrm{R}^{2}$ value. The $\mathrm{n}$ value in Korsmeyer-Peppas indicates the type of drug release mechanism. According to a review from Dash et al. (2010), n value below 0.5 indicates Fickian diffusion whereas $\mathrm{n}$ value greater than 0.5 indicates nonFickian mechanism of drug release. In condition of $n$ value equal 
to 0.89 , it indicates Case II transport and when $\mathrm{n}$ value greater than 0.89 , it indicates super case II transport.

\section{RESULTS AND DISCUSSION}

\section{Characterization of Formulated Nanosuspensions}

The 15 formulated nanosuspensions were evaluated based on particle size, PDI, and zeta potential by using Malvern Zetasizer. The result of particle size, PDI and zeta potential of formulated nanosuspensions are shown in Table 2.

Table 2: Particle size, PDI and Zeta potential of formulated nanosuspensions

\begin{tabular}{cccc}
\hline Formulation & $\begin{array}{c}\text { Particle Size } \\
(\mathbf{n m})\end{array}$ & PDI & $\begin{array}{c}\text { Zeta Potential } \\
(\mathbf{m V})\end{array}$ \\
\hline F1 & $764.5 \pm 209.1$ & 0.419 & -9.52 \\
F2 & $554.1 \pm 98.68$ & 0.580 & -8.57 \\
F3 & $752.0 \pm 239.6$ & 0.522 & -7.58 \\
F4 & $509.0 \pm 319.2$ & 0.428 & -9.81 \\
F5 & $488.5 \pm 271.7$ & 0.660 & -8.41 \\
F6 & $500.4 \pm 98.71$ & 0.599 & -10.00 \\
F7 & $801.0 \pm 193.3$ & 0.421 & -8.13 \\
F8 & $614.1 \pm 197.6$ & 0.529 & -8.55 \\
F9 & $774.6 \pm 213$ & 0.431 & -10.00 \\
F10 & $836.6 \pm 321.3$ & 0.547 & -5.04 \\
F11 & $588.1 \pm 168$ & 0.520 & -8.43 \\
F12 & $583.9 \pm 207.4$ & 0.542 & -8.12 \\
F13 & $539.8 \pm 240$ & 0.595 & -6.92 \\
F14 & $593.5 \pm 214.3$ & 0.526 & -9.08 \\
F15 & $695.9 \pm 19.05$ & 0.619 & -8.57 \\
\hline
\end{tabular}

Basically, all 15 formulations had particle size within the nanosize range $400 \mathrm{~nm}$ to $900 \mathrm{~nm}$. Formulation F5 that produced under $30 \% \mathrm{w} / \mathrm{w}$ stabilizer:drug mass ratio, 1300 bar in first 10 homogenization cycles and 15 cycles for 1500 bar of homogenization pressure had shown the smallest particle size with $488.5 \mathrm{~nm}$. Formulation F7 with $30 \%$ w/w stabilizer:drug mass ratio, 800 bar in first 10 homogenization cycles and 5 cycles for 1500 bar of homogenization pressure had shown the greatest particle size with $801 \mathrm{~nm}$. It was found that the increase in homogenization pressure and homogenization cycle resulted in smaller particle size. When there is increment of pressure and cycle in homogenization, it provides greater particles collision and higher shear force in cavitation for fining down the particles (Yadav and Singh, 2012).

According to the PDI study (Table 4.5), most of the formulations obtained value above 0.5 , it indicates non-uniformity distribution of particles in nanosuspension. Formulations F1 (0.419), F4 (0.428), F7 (0.421) and F9 (0.431) obtained PDI below 0.5 which is acceptable in term of uniformity of particles distribution. Ideally, PDI value in between 0.1 to 0.25 is required to achieve better physical stability with the enhanced uniformity of particles distribution (Sabeti et al., 2014).Thus, stabilizer type and concentration need to be researched further to develop the appropriate formulation with improved distribution.

Zeta potential analysis was carried out to study surface characteristics of nanosuspension in order to indicate the stability behaviour of each formulation. Generally, a more negative value than $-30 \mathrm{mV}$ or more positive value than $+30 \mathrm{mV}$ is required to obtain better stability of nanosuspension (Honary and Zahir,
2013). However, all formulations prepared have shown less negative zeta potential which indicates the instability of formulated nanosuspension. Then, in order to enhance the stability, further addition of ionic agent is suggested (Doymus, 2007).

\section{Response Analysis and Response Surface Analysis}

Based on the $\mathrm{R}^{2}$ obtained for each response, it shown that particle size is the most affected response by the change in the parameters. Particle size also is the only response that fitted to the model as the $\mathrm{R}^{2}$ value $(93.15 \%)$ had exceeded $90 \%$ which indicates the variables are very highly correlated. Both PDI and zeta potential had $\mathrm{R}^{2}$ below $80 \%$ which means that these 2 responses are not well fitted to the model (Table 3) Thus, the response optimizer was manipulated based on particle size only.

An equation was established based on the RSM analysis as stated in Figure 3.16 on particle size:

$Y=597.167-18.137 \mathrm{X}_{1}-121.913 \mathrm{X}_{2}-77.25 \mathrm{X}_{3}+19.579 \mathrm{X}_{1}^{2}+$ $28.079 \mathrm{X}_{2}^{2}+32.154 \mathrm{X}_{3}-7.325 \mathrm{X}_{1} \mathrm{X}_{2}+46.45 \mathrm{X}_{1} \mathrm{X}_{3}-12.65 \mathrm{X}_{2} \mathrm{X}_{3}$ where $\mathrm{Y}$ indicated particle size; $\mathrm{X}_{1}$ indicated stabilizer:drug mass ratio; $\mathrm{X}_{2}$ indicated homogenization pressure in first 10 cycles; $\mathrm{X}_{3}$ indicated number of remaining homogenization cycle under 1500 bar.

Based on Figure 1, the parameters that significantly affected the particle size of nanosuspension $(\mathrm{p}<0.05)$ include homogenization pressure in first 10 cycles with p-value of 0.001 and number of remaining homogenization cycle under 1500 bar with p-value of 0.009 . stabilizer:drug mass ratio had p-value of 0.372 which indicates this parameter is not significantly affecting the particle size. The surface plot shown in Figure 2 and Figure 3 provided additional evidence to support insignificant effect of stabilizer:drug mass on particle size.

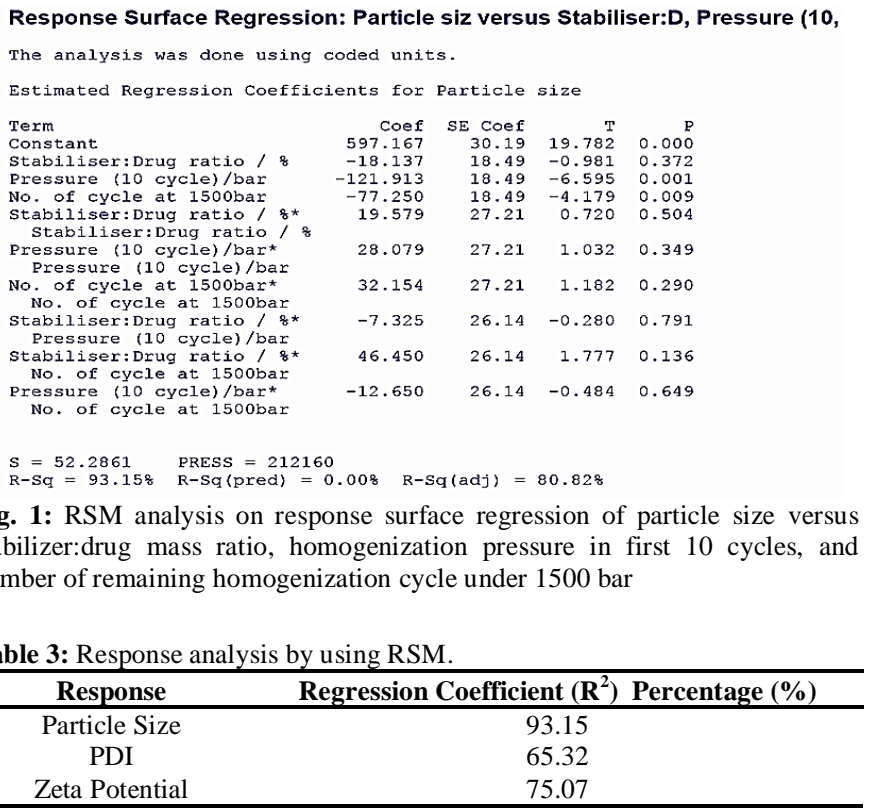




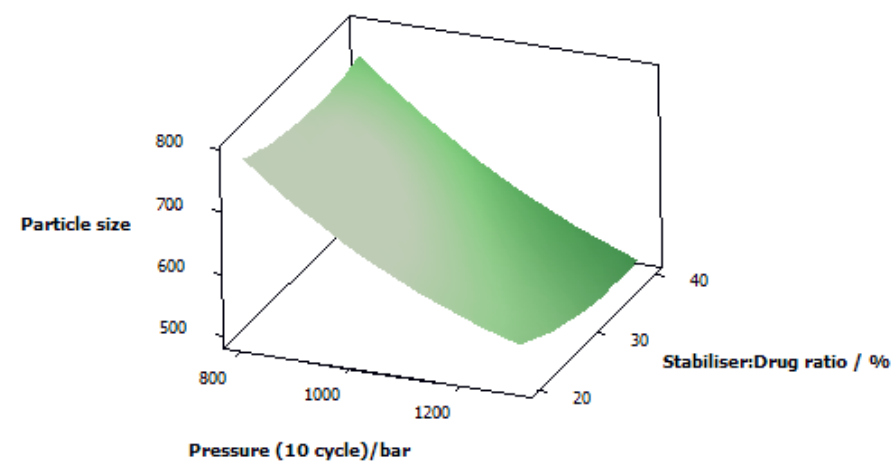

Fig. 2: Surface plot of particle size versus homogenization pressure in first 10 cycles and stabilizer:drug mass ratio.

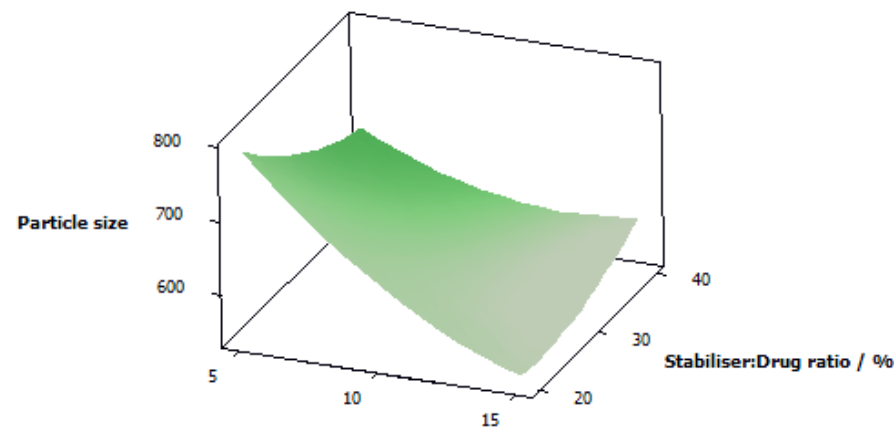

No. of remaining cycles at 1500 bar

Fig. 3: Surface plot of particle size versus stabilizer:drug mass ratio and number of remaining homogenization cycle under 1500 bar.

Based on Figure 2, the surface plot shows that the increase of homogenization pressure in first 10 cycles able to reduce the particle size of drug proportionally whereas the increase of stabilizer:drug mass ratio didn't shown much effect in particle size reduction. According to Figure 18, the increase of number of remaining homogenization cycle under 1500 bar also able to reduce the particle size proportionally. However, the particle size became slightly bigger with the increase of stabilizer:drug mass ratio instead of giving reduction effect which may due excessive envelop of stabilizer surround the drug particles (Afifi et al., 2015). In Figure 4, it shows that the increase of both homogenization pressure in first 10 cycles and number of remaining homogenization cycle under 1500 bar gave combination effect in the particle size reduction.

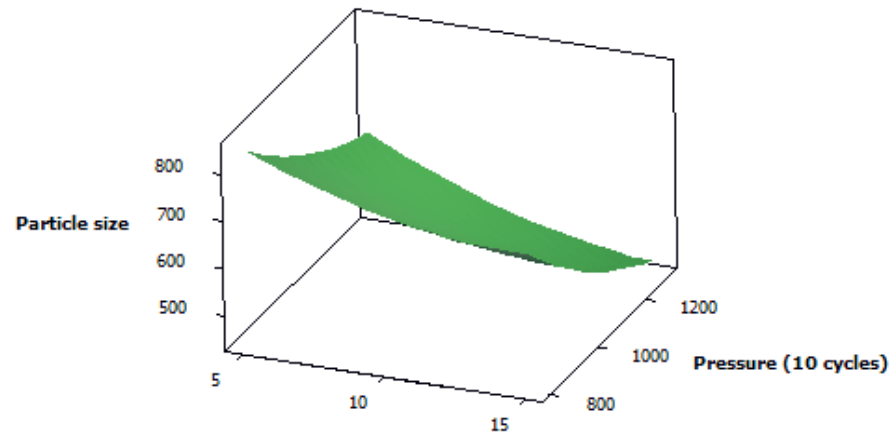

No. of remaining cycles at $1500 \mathrm{bar}$

Fig. 4: Surface plot of particle size versus homogenization pressure in first 10 cycles and number of remaining homogenization cycle under 1500 bar.

\section{Optimization of Process and Formulation by RSM}

The study was continued with the optimization of the process and formulation parameters by using response optimizer of RSM to select 3 formulations. The selected formulations were evaluated on the basis of drug content, DEE, and in vitro dissolution study for selection of the most optimized formulation in present study.

The selection of formulations was based on 3 different predicted particle size in order to check the influence of particle size of drug on the dissolution rate. Thus, the formulation FF3 $(440 \mathrm{~nm})$ with smallest predicted particle size was selected and also predicted as the optimized formulation with expectation of better dissolution result due to its smaller particle size. In addition, another 2 formulations, formulation FF1 $(701.9 \mathrm{~nm})$ and formulation FF2 $(542.8 \mathrm{~nm})$ with bigger predicted particle size were selected for the purpose of comparison. These 3 formulations were prepared in according to the value of parameters shown in the response optimizer and the parameters values were listed in Table 4.

Table 4: Selected formulations based on response optimizer with the respective predicted particle size.

\begin{tabular}{|c|c|c|c|c|}
\hline 苞 & 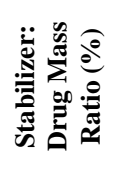 & 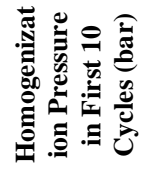 & 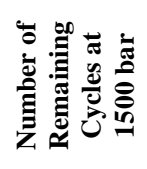 & 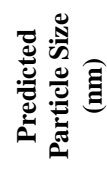 \\
\hline FF1 & 25 & 800 & 15 & 701.9 \\
\hline FF2 & 25 & 1050 & 15 & 542.8 \\
\hline FF3 & 25 & 1300 & 15 & 440.0 \\
\hline
\end{tabular}

The selected formulations were characterized on particle size, PDI and zeta potential and the results were depicted in Table 5. The observed particle size of selected formulations was compared with the predicted particle size as shown in Table 6 . The result shows that the observed values were quite closed to the predicted value as the percentage of bias was low, indicates the prediction of the response optimizer was trustworthy.

Table 5: Particle size, PDI and zeta potential of selected formulations.

\begin{tabular}{cccc}
\hline Formulation & Particle Size $(\mathbf{n m})$ & PDI & Zeta Potential $(\mathbf{m V})$ \\
\hline FF1 & $728.2 \pm 248.1$ & 0.456 & -9.06 \\
FF2 & $504.3 \pm 213.8$ & 0.625 & -5.05 \\
FF3 & $478.1 \pm 289.9$ & 0.730 & -6.61 \\
\hline
\end{tabular}

Table 6: Comparison of predicted and observed experimental values of particle size the nanosuspension of selected formulations.

\begin{tabular}{cccc}
\hline Formulation & $\begin{array}{c}\text { Predicted Particle } \\
\text { Size }(\mathbf{n m})\end{array}$ & $\begin{array}{c}\text { Particle Size } \\
(\mathbf{n m})\end{array}$ & $\begin{array}{c}\text { Percentage of } \\
\text { Bias (\%) }\end{array}$ \\
\hline FF1 & 701.9 & $728.2 \pm 248.1$ & -3.61 \\
FF2 & 542.8 & $504.3 \pm 213.8$ & 7.63 \\
FF3 & 440.0 & $478.1 \pm 289.9$ & -7.97 \\
\hline
\end{tabular}

\section{Evaluation of Selected Formulations}

The prepared formulations were sent for evaluation tests on drug content, DEE, and in vitro dissolution study. Drug content estimation study was carried out to determine the percentage of actual drug content in the nanosuspension prepared. Based on the 
results listed in Table 7. All selected formulations had very good yield which indicates there was minimum loss of drug in the process of nanosuspension production and most of the drug added was conserved in the formulations. The DEE results of nanosuspension selected were depicted in Table 8. All selected formulations have very high efficiency in drug entrapment.

Table 7: Drug content of nanosuspension in selected formulations.

\begin{tabular}{|c|c|c|c|c|}
\hline 营 & 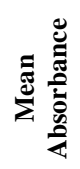 & 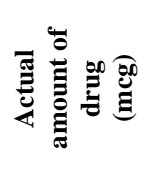 & 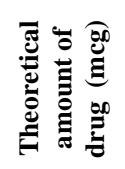 & 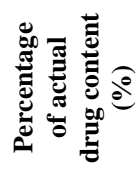 \\
\hline FF1 & 0.548 & 197.8 & 200 & 98.90 \\
\hline FF2 & 0.544 & 196.3 & 200 & 98.15 \\
\hline FF3 & 0.542 & 195.6 & 200 & 97.80 \\
\hline
\end{tabular}

Table 8: DEE of nanosuspension in selected formulations.

\begin{tabular}{|c|c|c|c|c|}
\hline 离 & 冚 & 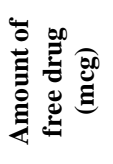 & 焉总䒿 & 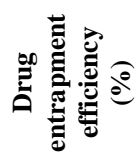 \\
\hline FF1 & 0.042 & 1.479 & 197.8 & 99.25 \\
\hline FF2 & 0.060 & 2.130 & 196.3 & 98.91 \\
\hline FF3 & 0.049 & 1.732 & 195.6 & 99.11 \\
\hline
\end{tabular}

In vitro dissolution study was carried out to evaluate the drug release rate of nanosuspension using dialysis bag. Dissolution study of the pure drug suspension and selected formulations was evaluated in two different dissolution media, includes $0.1 \mathrm{~N} \mathrm{HCl}$ and $\mathrm{pH} 6.8$ phosphate buffer with $0.1 \%$ w/v Tween 80 . The $\%$ CDR of selected formulations was compared with the pure drug suspension to check for the improvement in drug dissolution rate.

Based on Figure 5, the selected formulations in $0.1 \mathrm{~N} \mathrm{HCl}$ have shown abrupt increase in the drug release of almost $80 \%$ in the initial $10 \mathrm{~min}$ while the pure drug suspension, it took $20 \mathrm{~min}$ to reach peak drug release $(47.51 \%)$. By comparing the \% CDR of selected formulations with the pure drug suspension, nanosuspensions showed doubling-up effect in drug release compared to pure drug suspension in $0.1 \mathrm{~N} \mathrm{HCl}$. According to Figure 6, the selected formulations showed constant increase in drug release in phosphate buffer while for pure drug suspension, it also showed constant increase in drug release but in a slower manner throughout the 120 min dissolution test.

The nanosuspensions were found to have around 10-fold higher \% CDR compared to pure drug suspension in phosphate buffer. By referring both Figure 5 and 6, formulation FF2 found to have greater \% CDR than FF1 while FF3 had the highest \% CDR among the selected formulations attributed to the smallest particle size obtained with formulation FF3. It can be further explained that the finer the drug particles, the greater the surface area which can provide the enhanced wetting on drug particles surface for better dissolution and resulting in improved drug release of nanosuspensions (Liu et al., 2010). Thus, FF3 which had the smallest particle size $(478.1 \mathrm{~nm})$ and the highest \% CDR in both $0.1 \mathrm{~N} \mathrm{HCl} \mathrm{(98.37 \% )}$ and phosphate buffer (48.67\%) was confirmed as the optimized formulation as predicted.

However, nanosuspensions in $0.1 \mathrm{~N} \mathrm{HCl}$ showed greater $\%$ CDR than phosphate buffer with $0.1 \% \mathrm{w} / \mathrm{v}$ Tween 80 . Although Tween 80 was added in phosphate buffer to enhance solubility of clopidogrel in basic environment, the drug still showed comparatively better solubility and faster dissolution rate in $0.1 \mathrm{~N}$ $\mathrm{HCl}$. This behaviour indicates $\mathrm{pH}-d e p e n d e n t$ solubility of clopidogrel in acidic environment.

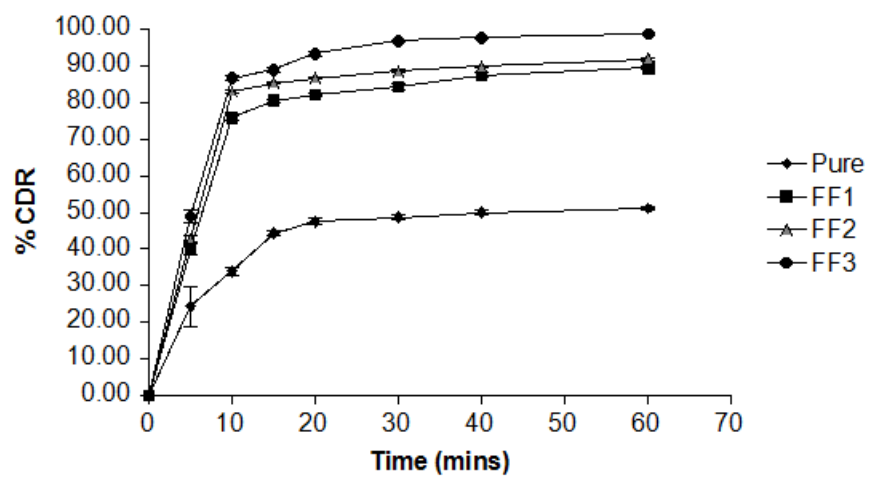

Fig. 5: Graph of percentage of CDR versus time for pure drug suspension, FF1, FF2 and FF3 nanosuspensions in $0.1 \mathrm{~N} \mathrm{HCl}$

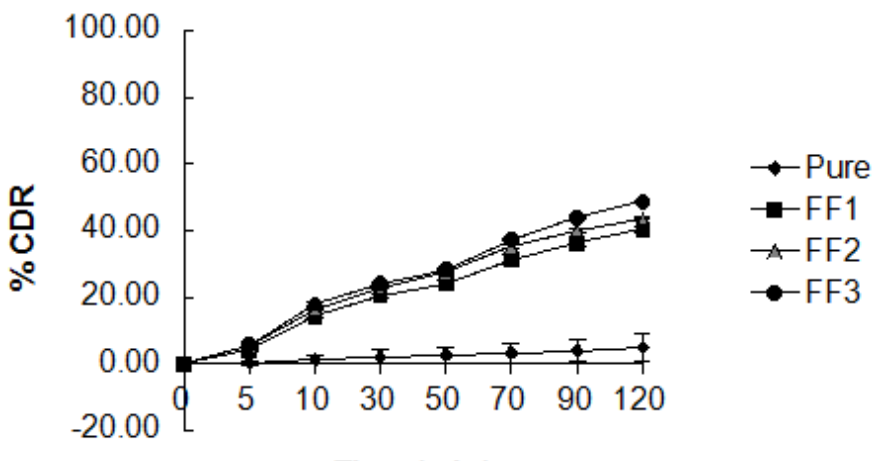

Time (min)

Fig. 6: Graph of percentage of CDR versus time for pure drug suspension, FF1, FF2 and FF3 nanosuspensions in pH 6.8 phosphate buffer with $0.1 \%$ w/v Tween 80 .

\section{Drug Release Kinetic Study of the Optimized Formulation}

Drug release kinetic study was carried out to describe the release behavior of nanosuspension of the optimized formulation (FF3) based on the dissolution profile in $0.1 \mathrm{~N} \mathrm{HCl}$ and $\mathrm{pH} 6.8$ phosphate buffer. The release kinetic study was evaluated according to the model parameters. The model of release kinetic study that had been carried out include zero order, first order, Higuchi model, and Korsmeyer-Peppas model of drug release. Graphs of release kinetic study were plotted for each model and showed in Figure 7, 8, 9, and 10 for $0.1 \mathrm{~N} \mathrm{HCl}$ while Figure 11, 12,13 and 14 were showed for phosphate buffer. 


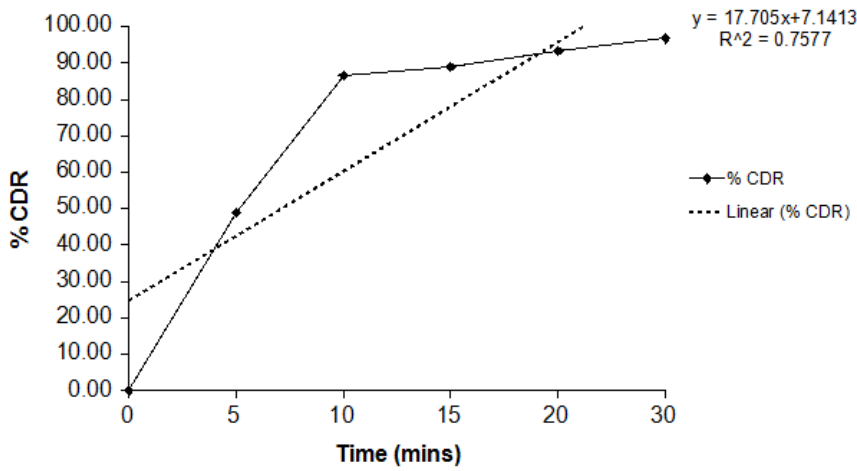

Fig. 7: Zero order release of clopidogrel nanosuspension formulation FF3 in $0.1 \mathrm{~N} \mathrm{HCl}$.

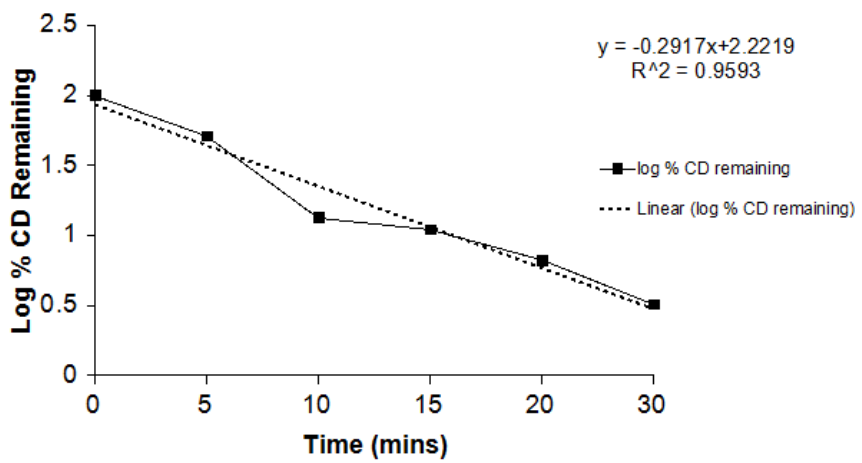

Fig. 8: First order release of clopidogrel nanosuspension formulation $\mathrm{FF} 3$ in $0.1 \mathrm{~N} \mathrm{HCl}$.

In $0.1 \mathrm{~N} \mathrm{HCl}$, the $\mathrm{R}^{2}$ value obtained was 0.7577 in zero order (Figure 22), 0.9593 in first order (Figure 8), 0.7577 in Higuchi model (Figure 9), and 0.6488 in Korsmeyer-Peppas model (Figure 10) which indicates the drug release in $0.1 \mathrm{~N} \mathrm{HCl}$ is best fitted with first order kinetic. First order kinetic states that the drug release is proportional to the concentration of drug remained (Dash et al., 2010). Thus, with the increase of dissolution time, the less concentration of drug remained in the dialysis bag, resulting in reduced rate of drug release after $10 \mathrm{~min}$ (Figure 9).

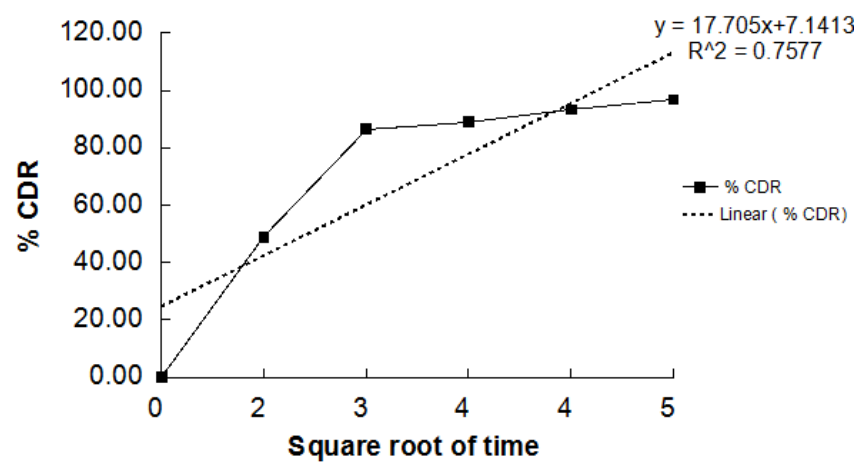

Fig. 9: Higuchi model release of clopidogrel nanosuspension formulation FF3 in $0.1 \mathrm{~N} \mathrm{HCl}$.

In phosphate buffer, the $\mathrm{R}^{2}$ value obtained was 0.9888 in zero order (Figure 11), 0.9930 in first order (Figure 12), 0.9888 in Higuchi model (Figure 13), and 0.8317 in Korsmeyer-Peppas model (Figure 14). The drug release in phosphate buffer is best fitted with first order as this model produced the highest value in $\mathrm{R}^{2}$ which indicates the concentration-dependent drug release. The $\mathrm{n}$ value was $0.1328 \quad(\mathrm{n}<0.5)$, indicates quasi-fickian diffusion behavior (Basak et al., 2008).

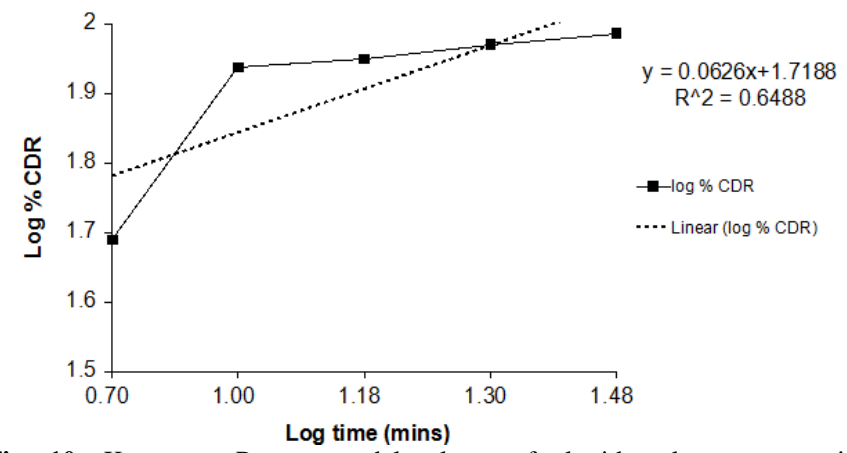

Fig. 10: Korsmeyer-Peppas model release of clopidogrel nanosuspension formulation $\mathrm{FF} 3$ in $0.1 \mathrm{~N} \mathrm{HCl}$.

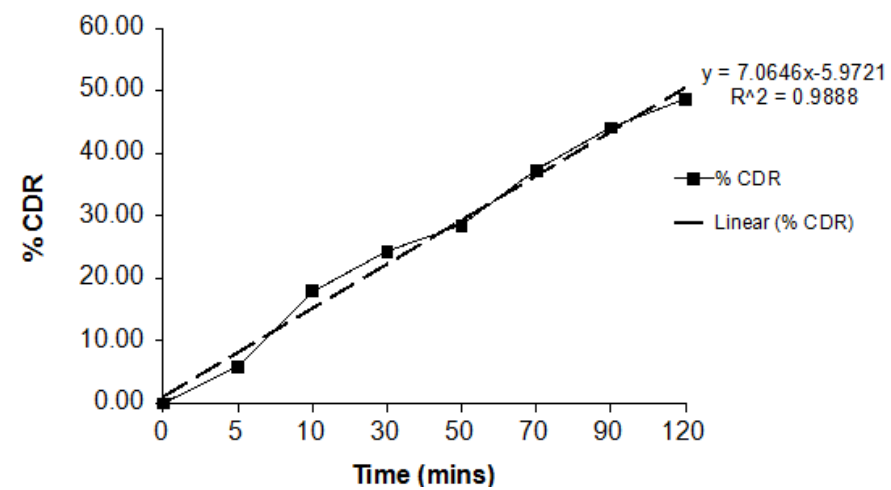

Fig. 11: Zero order release of clopidogrel nanosuspension formulation FF3 in pH 6.8 phosphate buffer with $0.1 \%$ w/v Tween 80 .

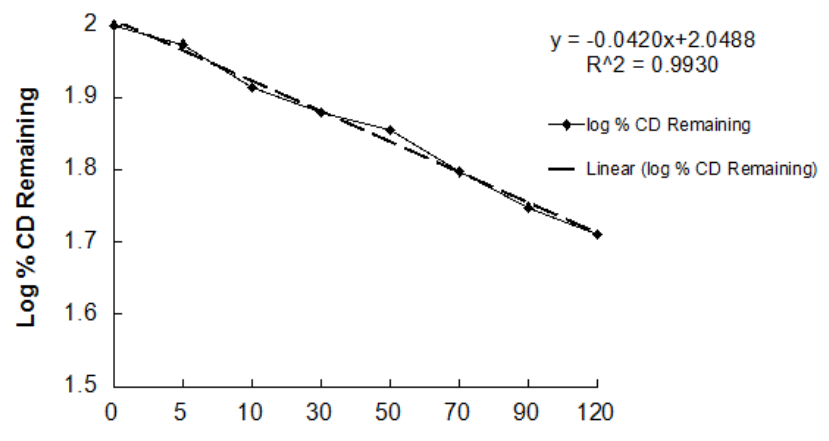

Time (mins)

Fig. 12: First order release of clopidogrel nanosuspension formulation FF3 in pH 6.8 phosphate buffer with $0.1 \%$ w/v Tween 80 .

\section{CONCLUSION}

High pressure homogenization method can be used as an effective tool for preparation of nanosized formulations. 
Clopidogrel nanosuspension prepared by this method showed significant improvement in aqueous solubility as well as dissolution characteristics which may significantly improve its oral bioavailability. Smaller particle size was obtained in formulations with higher homogenization pressure and greater homogenization cycle.

\section{ACNOWLEDGEMENT}

Authors are thankful to MAHSA University and School of Pharmacy, Taylor's University for supporting and providing the laboratory facilities for this project completion.

\section{Financial support and sponsorship: Nil.}

Conflict of Interests: There are no conflicts of interest.

\section{REFERENCES}

Afifi SA, Hassan MA, Abedlhammed AS and Elkhodairy KA. Nanosuspension: An emerging trend for bioavailability enhancement of etodolac, Int J of Poly Sci, 2015; 2015:1-16.

Agarwal V and Bajpai M. Preparation and optimization of esomeprazole nanosuspension using evaporative precipitationultrasonication. Trop J of Pharm Res, 2014; 13(4):497-503.

Attari Z, Bhandari A, Jagadish PC and Lewis S. Enhanced ex vivo intestinal absorption of olmesartan medoxomil nanosuspension: Preparation by combinative technology. Saudi Pharm Journal, 2015; 24:57-63.

Basak SC, Kumar KS and Ramalingam M. Design and release characteristics of sustained release tablet containing metformin $\mathrm{HCl}$. Braz J of Pharm Sci, 2008; 44(3):477-483.

Chen $\mathrm{H}$, Khemtong $\mathrm{C}$, Yang $\mathrm{X}$, Chang $\mathrm{X}$ and Gao J. Nanonization strategies for poorly water-soluble drugs. Drug Discov Today, 2011; 16(7-8):354-360.

Chiang PC, Ran YQ, Chou KJ, Cui Y and Wong $\mathrm{H}$. Investigation of utilization of nanosuspension formulation to enhance exposure of 1,3-dicyclohexylurea in rats: Preparation for PK/PD study via subcutaneous route of nanosuspension drug delivery. Nanoscale Res Letters, 2011; 6: 413.

Dash S, Murthy PN, Nath L and Chowdhury P. Review: Kinetic Modeling on drug release from controlled drug delivery systems. Acta Polon Pharma Drug Res, 2010; 67(3):217-223.

Doymus K. The effect of ionic electrolytes and $\mathrm{pH}$ on the zeta potential of fine coal particles. Turk J of Chem, 2007; 31:589-597.

Honary S and Zahir F. Effect of zeta potential on the properties of nano-drug delivery systems- a review (part 2). Trop J of Pharm Res, 2013; 12(2): 265-273
Jagdale DM, Kamble VA and Kadam VJ. Nanosuspension a novel drug delivery system. Int J of Pharm and Bio Sci, 2010; 1(4): 352360 .

Kumar P and Singh C. A study on solubility enhancement methods for poorly water soluble drugs. The American J of Pharmacol Sci, 2013; 1(4):67-73.

Liu Y, Sun CS, Jiang TY, Zheng L and Wang SL. Mechanism of Dissolution Enhancement and Bioavailability of Poorly Water Soluble Celecoxib by Preparing Stable Amorphous Nanoparticles. J of Phar and Pharm Sci, 2010; 13(4): 589-606.

Mane AN, Gilda SS, Ghadge AA, Bhosekar AR and Bhosale RR. Nanosuspension - A novel carrier for lipidic drug transfer. Scholar Academic Journal of Pharm. 2014; 3(1):82-88.

Patel VR and Agrawal YK. Nanosuspension: An approach to enhance solubility of drugs, J Adv Pharm Tech and Res, 2011; 2(2):81-87.

Rabinow B.E. Nanosuspensions in drug delivery. Nat Rev Drug Disc, 2004; 3:785-796.

Raghuvanshi S and Pathak K. Recent advances in delivery systems and therapeutics of clopidogrel: A poorly water soluble drug with absorption window in stomach. J of Drug Del, 2014; 1-15.

Sabeti B, Noordin MI, Mohd S, Hashim R, Dahlan A and Javar HA. Development and characterization of liposomal doxorubicin hydrochloride with palm oil. Biomed Res Int, 2014; 1-6.

Shahba AAW, Mohsin K and Alanazi FK. Novel selfnanoemulsifying drug delivery systems (SNEDDS) for oral delivery of clopidogrel: design, optimization, and in-vitro assessment. AAPS Pharm SciTech, 2012; 13(3): 967-977.

Shid RL, Dhole SN, Kulkarni N and Shid SL. Nanosuspension: A Review. Int J Pharm Sci Rev and Res, 2013; 22(1):98-106.

Takagi TRC, Bermejo M, Yamashita S, Yu LX and Amidon GL. A provisional biopharmaceutical classification of the top 200 oral drug products in the United States, Great Britain, Spain, and Japan. Mol. Pharma, 2006; 3: 631-643.

Teva Pharmaceuticals Europe B.V. 2015. Package Leaflet: Clopidogrel 15 mg Tablets, UK, Ver. 1.6, 1-2.

Turner D, Lurie Y, Finkelstein Y, Schmid T, Gopher A, Kleid D and Bentur Y. Pediatric Clopidogrel Overdose and Toxicokinetics, AAP Journals, 2006; 117(5).

Yadav G and Singh SR. Nanosuspension: A promising drug delivery system, Pharmacophore, 2012; 3(5): 217-243.

\section{How to cite this article:}

Qureshi MJ, Phin FF, Patro S. Enhanced Solubility and Dissolution Rate of Clopidogrel Nanosuspension: Formulation via High Pressure Homogenization Technique and Optimization Using Box Behnken Design Response Surface Methodology. J App Pharm Sci, 2017; 7 (02): 106-113. 